\title{
Topology optimization of microwave waveguide filters
}

\author{
Aage, Niels; Johansen, Villads Egede
}

Published in:

International Journal for Numerical Methods in Engineering

Link to article, DOI:

10.1002/nme.5551

Publication date:

2017

Document Version

Peer reviewed version

Link back to DTU Orbit

Citation (APA):

Aage, N., \& Johansen, V. E. (2017). Topology optimization of microwave waveguide filters. International Journal for Numerical Methods in Engineering, 112(3), 283-300 . https://doi.org/10.1002/nme.5551

\section{General rights}

Copyright and moral rights for the publications made accessible in the public portal are retained by the authors and/or other copyright owners and it is a condition of accessing publications that users recognise and abide by the legal requirements associated with these rights.

- Users may download and print one copy of any publication from the public portal for the purpose of private study or research.

- You may not further distribute the material or use it for any profit-making activity or commercial gain

- You may freely distribute the URL identifying the publication in the public portal

If you believe that this document breaches copyright please contact us providing details, and we will remove access to the work immediately and investigate your claim. 


\section{Topology optimization of microwave waveguide filters}<smiles>[CH]1CCC1</smiles>

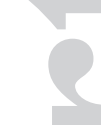

N. Aage ${ }^{1 * *}$ and V. Egede Johansen ${ }^{1,2 *}$

${ }^{1}$ Department of Mechanical Engineering, Solid Mechanics, Centre for Acoustic-Mechanical Micro Systems, Technical University of Denmark, DK-2800 Kgs. Lyngby.

${ }^{2}$ Department of Chemistry, University of Cambridge, Lensfield Rd., Cambridge, CB21EW, UK

We present a density based topology optimization approach for the design of metallic microwave insert filters. A two-phase optimization procedure is proposed in which we, starting from a uniform design, first optimize to obtain a set of spectral varying resonators followed by a band gap optimization for the desired filter characteristics. This is illustrated through numerical experiments and comparison to a standard band pass filter design. It is seen that the carefully optimized topologies can sharpen the filter characteristics and improve performance. Furthermore, the obtained designs share little resemblance to standard filter layouts and hence the proposed design method offers a new design tool in microwave engineering. Copyright (c) 2017 John Wiley \& Sons, Ltd.

Received ..

KEY WORDS: microwave waveguides, topology optimization, metallic design interpolation, electromagnetic waves, finite elements.

*The authors have contributed equally to this work.

${ }^{*}$ Correspondence to: Department of Mechanical Engineering, Solid Mechanics, Centre for Acoustic-Mechanical Micro Systems, Technical University of Denmark, Bld. 404, DK-2800 Kgs. Lyngby. E-mail: naage @ mek.dtu.dk

This article has been accepted for publication and undergone full peer review but has not been through the copyediting, typesetting, pagination and proofreading process, which may lead to differences between this version and the Version of Record. Please cite this article as doi: $10.1002 / \mathrm{nme} .5551$ 


\section{INTRODUCTION}

Microwave filters are crucial to modern day telecommunication, sensing and other high technology applications. Ever since Marconi’s (wideband) spark-gap transmitters were replaced by continuous wave transmitters and the radio frequency spectrum started to crowd, microwave filters have been key in improving receiver performance and avoiding signal leaking at unwanted frequencies.

One class of microwave filters are made to fit metallic waveguides. Metallic waveguides are tubelike structures - most often of rectangular cross-section - inside which waves can propagate. They are low-loss and therefore suitable for a palette of applications ranging from extremely sensitive systems (like space communication) or high energy systems where losses would lead to heating or significant energy waste (radars, RF based particle accelerators, microwave ovens).

Waveguide filter design normally consists of designing a prototype filter network and then cascading inductive, capacitive or resonating elements to implement the prototype filter [1]. Some elements extend the geometry of the waveguide by coupling the waveguide to external resonators or stubs. Other elements are placed inside the existing waveguide geometry, like so-called post filters, iris coupled filters, dielectric resonators and insert filters. These filter design elements are usually based on structures to which an approximate analytical model exists, such that the behavior can be shaped to fit the prototype filter elements. This enables realization of almost any desired filter, but often at the practical cost of vast space/weight consumption due to cascading of elements.

To enable more compact, high-performance filters, this work proposes a topology optimization approach to filter design. Such approach has the clear advantage of being able to design one integrated structure to obtain a given set of filter properties instead of a cascaded series of elements. Former work on synthesis of waveguide filters using topology optimization has mainly focused on dielectric materials $[2,3,4,5,6]$ which can be fabricated using e.g. additive manufacturing techniques [7]. Sharper filter responses can in general be obtained using metallic structures due to the large metal/air contrast. and since the only loss induced is due to the penetration depth of metal. At the same time, topology optimization of metallic structures is a much more difficult problem. 
That is, for metallic structures, the electromagnetic fields are highly localized at the surface of the conductors. In terms of gradient based optimization this results in sensitivity fields that are also confined to the vicinity of the conductor. This, and the fact that electromagnetic design problems have highly oscillatory system responses $[8,9]$, means that brute-force approaches to metallic microwave topology optimization often leads to poor local minima and ill performing devices. These shortcomings can sometimes be circumvented by introducing artificial damping [8]. However, in the case of metallic microwave design problems, the material to be distributed is in itself a damping media. and hence the artificial damping is inadequate. Instead this class of design problems can be solved utilizing a filtering scheme $[10,11,12,13]$.

The only successful attempt on topology optimization of metallic waveguide filters known by the authors is by [14], but here a genetic algorithm was used which strongly limits its application to larger problems. Other attempts on metallic micro strip resonator design also exists [15] but no usable designs were obtained. Interest in research on metallic microwave optimization is increasing $[10,13,16,17,18]$ and the methods have been applied several times to antenna design problems. Using experience and knowledge gained through these works, we are at a state where attempting to design usable metallic waveguide filters is a feasible task.

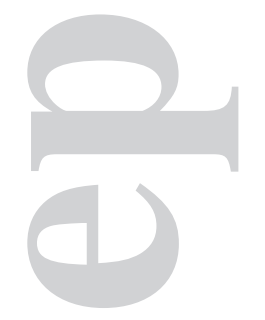

\section{PHYSICAL MODEL}

The model problem to be investigated is illustrated in Fig. 1. The figure shows a section of a rectangular waveguide in which we need to solve Maxwell's equations along with appropriate boundary conditions $[19,20]$. In this work we use linear, isotropic materials with no free charges and solve the problem in the frequency domain. This covers most materials used in passive, metallic microwave devices. Maxwell's equations are therefore conveniently cast as a single second order Partial Differential Equation (PDE) in the electric field as follows

$$
\nabla \times\left(\mu_{r}^{-1} \nabla \times \boldsymbol{E}\right)-k_{0}^{2}\left(\epsilon_{r}-j \frac{\sigma}{\omega \epsilon_{0}}\right) \boldsymbol{E}=0 \text { in } \Omega,
$$

This article is protected by copyright. All rights reserved. 

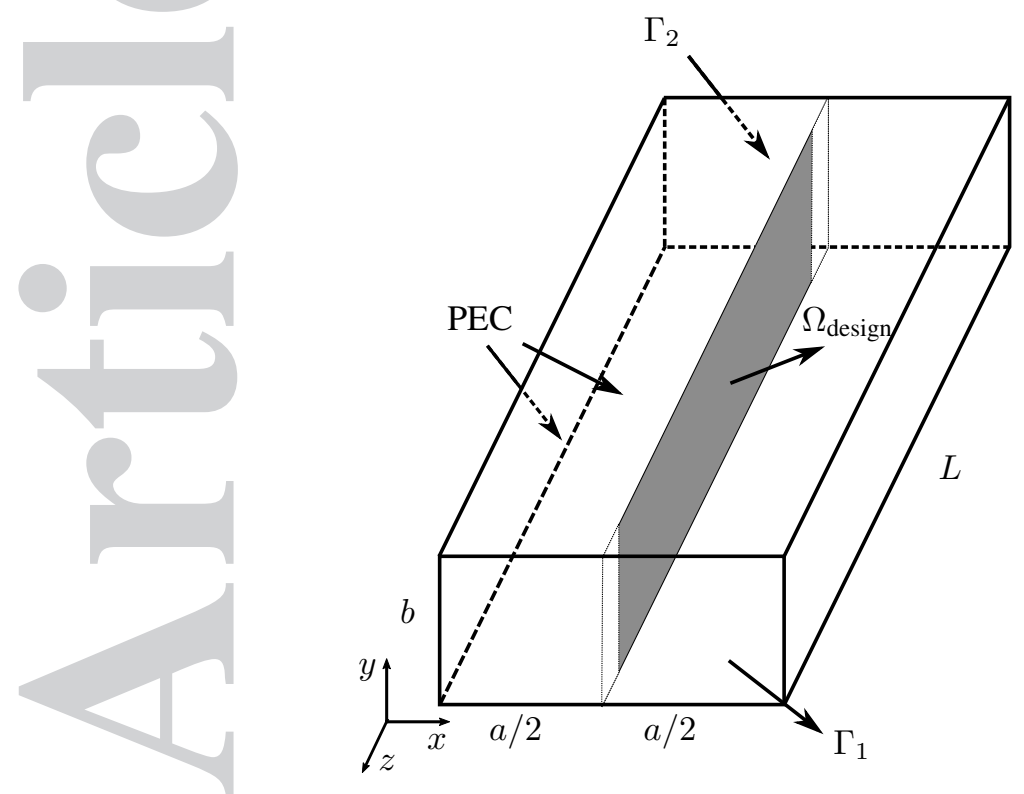

Figure 1. Basic geometry for the rectangular waveguide problem (WR-90) with $a=22 \mathrm{~mm}$ and $b=10 \mathrm{~mm}$. The boundary condition, i.e. PEC and waveguide ports can be seen as well as the design domain for the optimization process, here denoted with $\Omega_{\text {design }}$. For the model problem examined in this paper, the length of the design domain is $32.6 \mathrm{~mm}$ and the total length of the computational domain is $42.6 \mathrm{~mm}$.

where $\boldsymbol{E}=\boldsymbol{E}(\boldsymbol{x})$ is the unknown complex electric field, $\omega$ is the frequency, $\mu_{r}$ is the relative permeability, $\epsilon_{r}$ the relative permittivity, $\sigma$ is the conductivity and $k_{0}=\omega \sqrt{\epsilon_{0} \mu_{0}}$ is the free space wave number. The two remaining constants are the free space permittivity $\epsilon_{0} \approx 8.8542 \cdot 10^{-12} \mathrm{~F} / \mathrm{m}$ and the free space permeability $\mu_{0}=4 \pi 10^{-7} \mathrm{H} / \mathrm{m}$. The real, physical solution can then be obtained from $\boldsymbol{E}_{\text {phys }}(\boldsymbol{x}, t)=\mathbb{R}\left(\boldsymbol{E}(\boldsymbol{x}) e^{j \omega t}\right)$.

The vector wave equation from Eq. (1) is solved for the following boundary conditions in the case of a metallic waveguide. The four sides that encloses the waveguide are modeled as Perfect Electric Conductors (PEC) which means that the tangential component of the electric field is zero, i.e.

$$
\boldsymbol{n} \times \boldsymbol{E}=\mathbf{0} \text { on } \Gamma_{\mathrm{PEC}},
$$

where $\boldsymbol{n}$ is an outward normal to the respective surfaces. The input and output ports, denoted with $\Gamma_{1}$ and $\Gamma_{2}$ in Fig. 1, are modeled using a waveguide port boundary condition [20]. This boundary condition utilizes that only certain modes can propagate in a given waveguide geometry and as a consequence these modes can be used to excite and absorb the electric field passing through 
the boundary. The modes can be solved analytically for simple geometries, or numerically for complicated port geometries. For the rectangular waveguide the first transverse electric mode, $\mathrm{TE}_{10}$,

is

$$
\boldsymbol{e}_{10}^{\mathrm{TE}}(\boldsymbol{x})=-\sqrt{\frac{2}{a b}}\left\{\begin{array}{c}
0 \\
\sin (\pi x / a) \\
0
\end{array}\right\},
$$

when the port is situated in the $x y$-plane (i.e. the wave is propagating along the $z$-axis). Note that it is common practice to operate waveguides using only one mode. Now the boundary condition for the input and output port can be stated as

$$
\begin{array}{ll}
\boldsymbol{E}=\boldsymbol{E}_{\mathrm{inc}}+c_{10,1} \boldsymbol{e}_{10}^{\mathrm{TE}} e^{j \gamma z} & \text { on } \Gamma_{1}, \\
\boldsymbol{E}=c_{10,2} \boldsymbol{e}_{10}^{\mathrm{TE}} e^{j \gamma z} & \text { on } \Gamma_{2},
\end{array}
$$

where $\boldsymbol{E}_{\text {inc }}$ is the incident field, $\gamma=\sqrt{k_{z}^{2}-k_{0}^{2}}$ is the propagation constant and $k_{z}=\pi / a$ is the port cutoff wave number. The constants $c_{10, i}$ can be determined from orthogonality. The latter yields the following

$$
\begin{aligned}
& c_{10,1}=e^{-j \gamma z} \int_{\Gamma_{1}} \boldsymbol{e}_{10}^{\mathrm{TE}} \cdot\left(\boldsymbol{E}-\boldsymbol{E}_{\mathrm{inc}}\right) \mathrm{d} \Gamma \\
& c_{10,2}=e^{-j \gamma z} \int_{\Gamma_{2}} \boldsymbol{e}_{10}^{\mathrm{TE}} \cdot \boldsymbol{E} \mathrm{d} \Gamma .
\end{aligned}
$$

These expressions are now re-inserted into Eqs. (4) and (5) after which the natural (Neumann) operator for the Maxwell problem, i.e. $\boldsymbol{n} \times \nabla \times$, is applied. Furthermore, the incident wave is chosen as the first order mode for the waveguide, i.e. $\boldsymbol{E}_{\mathrm{inc}}=\boldsymbol{e}_{10}^{\mathrm{TE}}$. Combining all of the above and utilizing orthogonality, one obtain the following port boundary conditions for the waveguide problem

$$
\begin{array}{ll}
\boldsymbol{n} \times \nabla \times \boldsymbol{E}=j \gamma \boldsymbol{e}_{10}^{\mathrm{TE}} \cdot \int_{\Gamma_{1}} \boldsymbol{e}_{10}^{\mathrm{TE}} \cdot \boldsymbol{E} \mathrm{d} \Gamma-2 j \gamma \boldsymbol{e}_{10}^{\mathrm{TE}} & \text { on } \Gamma_{1}, \\
\boldsymbol{n} \times \nabla \times \boldsymbol{E}=j \gamma \boldsymbol{e}_{10}^{\mathrm{TE}} \cdot \int_{\Gamma_{2}} \boldsymbol{e}_{10}^{\mathrm{TE}} \cdot \boldsymbol{E} \mathrm{d} \Gamma & \text { on } \Gamma_{2},
\end{array}
$$


where the last term in Eq. (8) is recognized as the loading term due to the incident field. These conditions can naturally be incorporated into a variational form for the Maxwell PDE.

\section{FINITE ELEMENT FORMULATION AND IMPLEMENTATION}

The PDE problem described in Eqs. (1), (2), (8) and (9) leads to the following linear finite element formulation when using the standard Galerkin discretization approach [20]:

$$
\boldsymbol{S}(\omega) \boldsymbol{E}=\left(\boldsymbol{K}-\boldsymbol{M}+\boldsymbol{B}_{\Gamma_{1}}+\boldsymbol{B}_{\Gamma_{2}}\right) \boldsymbol{E}=\boldsymbol{f}(\omega),
$$

where the global system matrices and vectors are assembled from

$$
\begin{aligned}
\boldsymbol{K} & =\sum_{e} \int_{\Omega_{e}} \mu_{r}^{-1}\left(\nabla \times \boldsymbol{N}_{e}\right)^{T}\left(\nabla \times \boldsymbol{N}_{e}\right) \mathrm{d} \Omega \\
\boldsymbol{M} & =\sum_{e} \int_{\Omega_{e}} k_{0}^{2}\left(\epsilon_{r}-j \frac{\sigma}{\omega \epsilon_{0}}\right) \boldsymbol{N}_{e}^{T} \boldsymbol{N}_{e} \mathrm{~d} \Omega, \\
\boldsymbol{B}_{\Gamma_{i}} & =j \gamma \sum_{e} \int_{\Gamma_{i}} \boldsymbol{N}_{e}^{T} \boldsymbol{e}_{10}^{\mathrm{TE}} \mathrm{d} \Gamma \cdot \sum_{e} \int_{\Gamma_{i}}\left(\boldsymbol{e}_{10}^{\mathrm{TE}}\right)^{T} \boldsymbol{N}_{e} \mathrm{~d} \Gamma, i=1,2, \\
\boldsymbol{f} & =2 j \gamma \sum_{e} \int_{\Gamma_{1}} \boldsymbol{N}_{e}^{T} \boldsymbol{e}_{10}^{\mathrm{TE}} \mathrm{d} \Gamma
\end{aligned}
$$

where $\boldsymbol{N}(\boldsymbol{x})$ represent vector (Nedelec) element shape functions belonging to the Sobolev space $V:=H(\nabla \times \boldsymbol{v}, \Omega)=\left\{\boldsymbol{v} \in\left[L_{2}(\Omega)\right]^{3} \mid \nabla \times \boldsymbol{v} \in\left[L_{2}(\Omega)\right]^{3}\right\}$. In this work we use tetrahedral elements with zero/first order shape functions.

The finite element problem in Eqs. (10) - (14) is implemented in an in-house MPI based C++ code [21]. The meshing is performed in Cubit (Sandia National Laboratories) and the partitioning is done by METIS [22]. It is important to note that the outer product in Eq. (13) can result in a high degree of communication in the parallel implementation in case the port boundary is distributed which is due to the fact that the $\boldsymbol{B}$ matrices are dense. Therefore we require that each port is confined to a single partition as is illustrated in Fig. 2. The figure also indirectly shows the design domain as a

This article is protected by copyright. All rights reserved. 


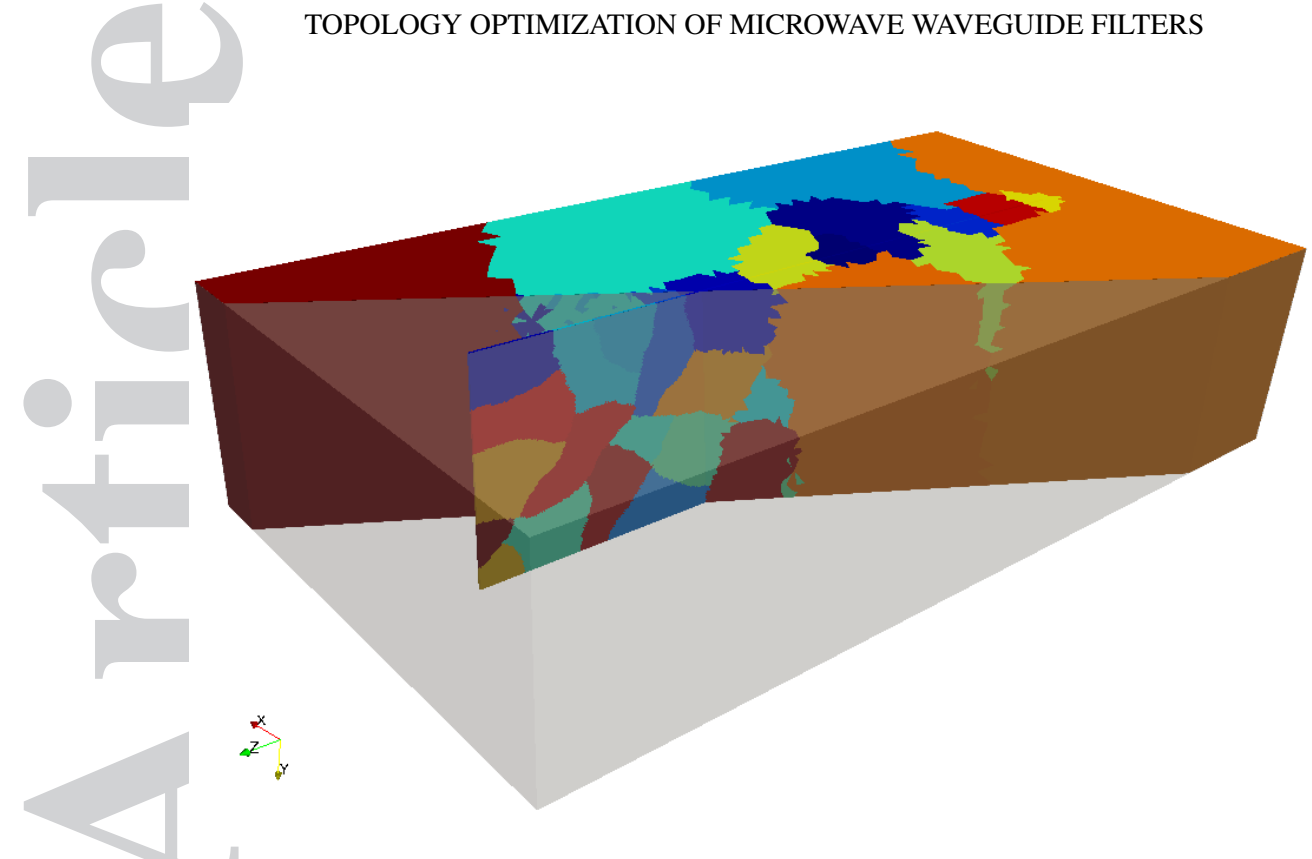

Figure 2. Partitioning for the waveguide problem. Note that the port is required to be on a single partition to facilitate easy construction of the outer product for the boundary integrals in Eq. (13). This can be seen by the orange partition to the right that fully encloses the 2 nd port surface. The thin plate in the center is the design domain.

thin plate which is partitioned heavily due to the fine mesh desired for a fine design representation.

The Maxwell problem is finally solved using the direct solver MUMPS [23].

\section{DESIGN INTERPOLATION}

The topology optimization methodology presented next falls into the category of so-called density based approaches [24]. That is, for each element in the design domain we assign a continuous variable, i.e. $\rho_{e} \in[0 ; 1]$ which we use to interpolate between two candidate materials. In the case of metallic waveguide filters, the relevant physical quantity is the conductivity. For details on electromagnetic theory the reader is referred to e.g. [19]. It is desired that the final design does not contain intermediate valued design variable such that the design is physically realizable. To obtain an interpolation scheme with such properties we adopt the scheme presented in [10]. For completeness the interpolation scheme is stated below.

This article is protected by copyright. All rights reserved. 
The design interpolation consists of two parts. The first interpolates directly in the material conductivity in the original Maxwell problem, i.e. Eq. (1) or (10) and can be written as

$$
\sigma(\rho)=\sigma_{0} 10\left[\log _{10}\left(\frac{\sigma_{d}}{\sigma_{0}}\right)+\rho\left\{\log _{10}\left(\frac{\sigma_{m}}{\sigma_{0}}\right)-\log _{10}\left(\frac{\sigma_{d}}{\sigma_{0}}\right)\right\}\right],
$$

where subscripts $m$ and $d$ refers to the metal and the background dielectric respectively, and where $\sigma_{0}$ is a scaling included to make the interpolation physically sound. In the work presented here we use $\sigma_{0}=\sigma_{m}=10^{6} \mathrm{~S} / \mathrm{m}$ and $\sigma_{d}=10^{-4} \mathrm{~S} / \mathrm{m}$. The given values are chosen for the following reasons. Due to numerical precision it can be shown that using a conductivity larger than $10^{6} \mathrm{~S} / \mathrm{m}$ for the metal does not change the response of the system significantly. Using a larger value (e.g. copper has $\sigma_{m}=5.998 \times 10^{7} \mathrm{~S} / \mathrm{m}$ ) will lead to intermediate design variables in the final design since the system performance does not benefit from an increase in the conductivity above the proposed upper bound. The same argument holds for the background material, i.e. choosing $\sigma_{d}$ too low leads to numerical instabilities, while a too large value results in an over damped response and hence an unphysical system response.

The second part of the interpolation scheme takes the skin depth issue into account. That is, the distance an electromagnetic wave propagates into a good conductor before being reduced by a factor of $e^{-1}$. Since the skin depth of a good conductor is several orders of magnitude smaller than the actual device dimensions, the finite element model cannot capture the rapid decay unless additional measures are taken. If this is not treated the skin depth effect will again result in a nonmonotonically behaving system response on meshes that resolve the geometry but not the skin depth. To circumvent this limitation we apply an impedance boundary condition on all faces of a design element. This leads to the following condition

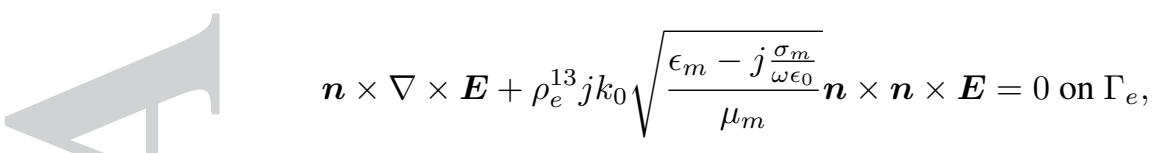

where $\Gamma_{e}$ refer to all faces of the $e$ 'th design element. From the two Eqs. (15) and (16) it is clear that $\rho_{e}=1$ yields a good conductor and that $\rho_{e}=0$ results in a background dielectric with very little This article is protected by copyright. All rights reserved. 
artificial damping. For further details on the interpolation scheme and its parameters the reader is referred to [10].

\section{OBJECTIVE FUNCTION}

The performance of a waveguide is often measure by its scattering, or $S$-parameters which provide information on the reflection and transmission of a wave in a waveguide. The complex $S$-parameters for a two port system can be computed as follows when only port 1 includes excitation:

$$
\begin{aligned}
& S_{11}(\omega)=\frac{\int_{\Gamma_{1}}\left(\boldsymbol{E}-\boldsymbol{E}_{\mathrm{inc}}\right) \cdot \overline{\boldsymbol{E}}_{\mathrm{inc}} \mathrm{d} \Gamma}{\int_{\Gamma_{1}}\left|\boldsymbol{E}_{\mathrm{inc}}\right|^{2} \mathrm{~d} \Gamma}, \\
& S_{21}(\omega)=\frac{\int_{\Gamma_{2}} \boldsymbol{E} \cdot \overline{\boldsymbol{E}}_{\mathrm{inc}} \mathrm{d} \Gamma}{\int_{\Gamma_{2}}\left|\boldsymbol{E}_{\mathrm{inc}}\right|^{2} \mathrm{~d} \Gamma},
\end{aligned}
$$

where $(\bar{\cdot})$ refers to complex conjugate and $S_{11}$ corresponds to the reflection at surface $\Gamma_{1}$ while $S_{21}$ corresponds to the transmission at $\Gamma_{2}$. A possible objective - or fitness - function for a given filter can be therefore be specified as a function of the $S$-parameters over some frequency range. To illustrate how this is to be done we use the pass band filter shown in Fig. 3. From the frequency sweep in Fig. $3 \mathrm{~b}$ it is clear that for frequencies lower than $9.8 \mathrm{GHz}$ and above $10.4 \mathrm{GHz}$, the transmission should be zero (or very small), while the transmission should be full, i.e. $\left|S_{21}\right|=1$, for the range between $9.8 \mathrm{GHz}$ and $10.4 \mathrm{GHz}$. We can state this formally in terms of a discrete frequency list and two index sets

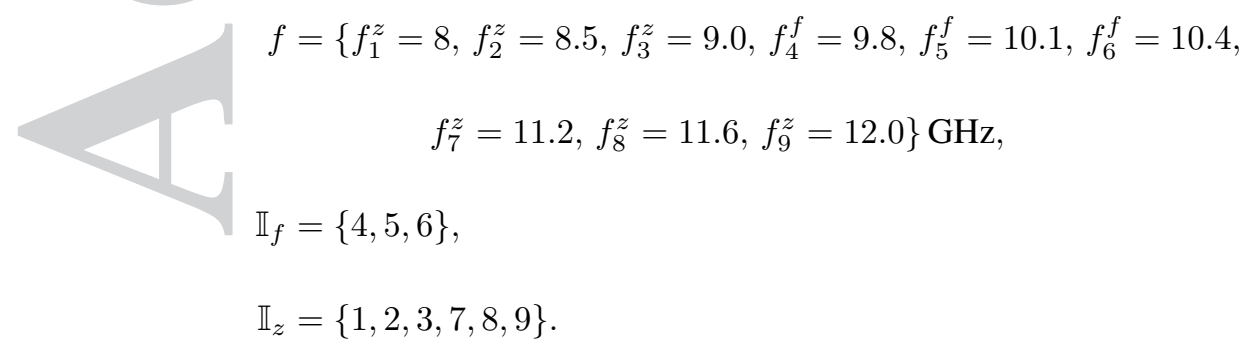

This article is protected by copyright. All rights reserved. 
where $f_{i}^{f}, \mathbb{I}_{f}$ and $f_{i}^{z}, \mathbb{I}_{z}$ refers to full and zero transmission respectively. The discrete frequency list can of course be refined or coarsened to fit a specific design task. The objective function can now be stated in terms of the transmission parameter as the following non-smooth maximization problem

$$
\Phi=\max \left[\min _{j \in \mathbb{I}_{f}}\left\{\left|S_{21}\left(\omega_{j}\right)\right|\right\}, \min _{i \in \mathbb{I}_{z}}\left\{1-\left|S_{21}\left(\omega_{i}\right)\right|\right\}\right]
$$

It is clear that $\Phi$ takes values between 0 and 1 and that $\Phi=1$ corresponds to the optimum. The non-smoothness can be remedied by introducing a bound formulation [24]. The bound formulation can in turn be stated in several different ways, which will be discussed in the following section.

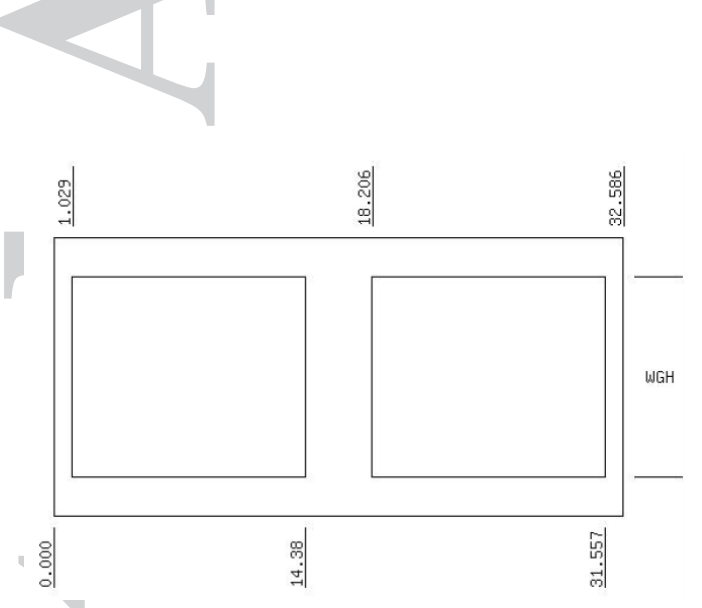

(a)

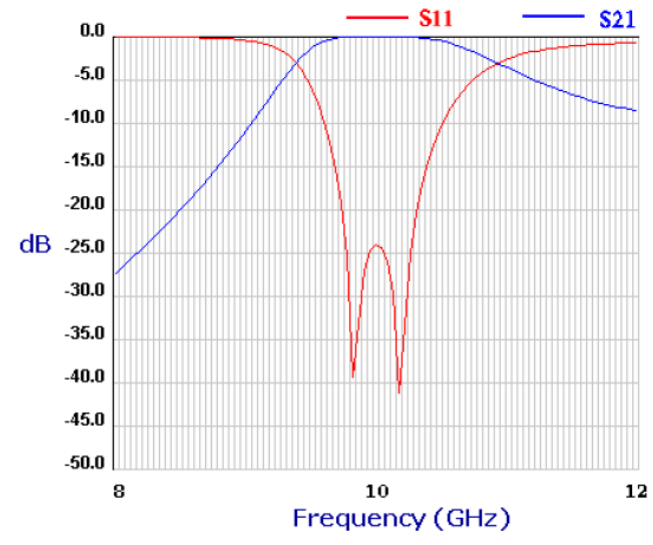

(b)

Figure 3. Screen shots from a 2-pole metal insert waveguide filter design obtained through www. guidedwavetech. com in 2015 (the tool is now taken off line). (a) Dimensions in mm of metallic structure to be inserted in the waveguide. Only the part contained within WGH (set to $10 \mathrm{~mm}$ ) is relevant. (b) filter response of the design. Since the company lives off selling their software, we find the example meets today's industry standards albeit real applications normally require more complex characteristics, but may be based on the same design principle.

\section{TWO PHASE OPTIMIZATION METHODOLOGY}

Even if a microwave filter is not based on cascading filter elements, it is unlikely to obtain good filter characteristics without a multipole design. From experience we have not been able to generate multipole design starting from a non-resonating starting guess since the optimization tends to get stuck in a local minimum containing only one pole. In recognition of this issue and the fact that we 
require our design methodology to be able to start from a uniform initial design, we have divided the optimization procedure in two phases: Phase 1 consists of designing a set of smaller resonators which can be used as initial guess for the full filter optimization, thus (over)populating the initial guess with several resonances. Phase 2 then consists of designing one integrated multipole structure that make up the overall filter response we are seeking.

The Phase 1 design problem(s), i.e. the resonator design, can be done in several ways using topology optimization. One way would be to do an eigenvalue target optimization where the difference between the resonance frequency and a target frequency is optimized (see e.g. [25]). This approach requires the solution of a quadratic eigenvalue problem due to the system damping, i.e. conductivity, which is currently not available within the used numerical framework. We therefore choose another design methodology capable of obtaining resonator designs which is based on the $S$-parameters and the non-smooth objective in Eq. (22). Restating this as a bound formulation we obtain the following smooth optimization problem

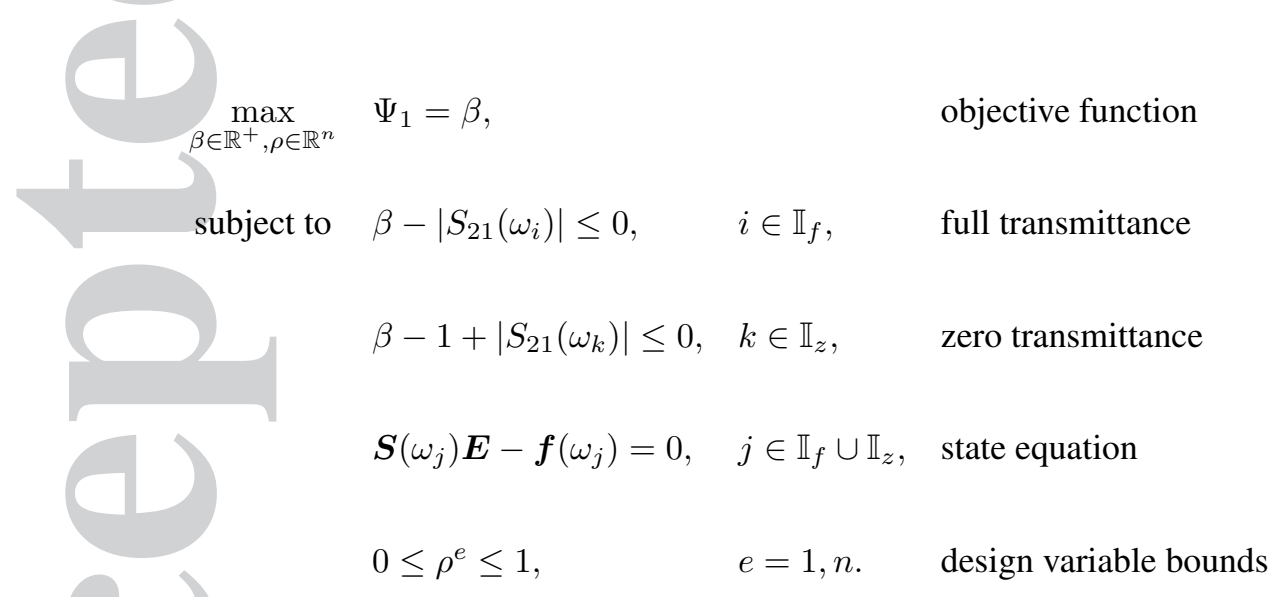

Note that the extra variable $\beta$ is required to transform the objective function from Eq. (22) into a smooth optimization problem that is tractable by standard gradient methods. Dependent on the specific filter design task, the number of needed Phase 1 problems will change and note that all Phase 1 problems are completely decoupled and can thus be solved completely independent of each other, i.e. they are embarrassingly parallel. It is an important observation that the formulation in Eq. (23) puts equal weight on transmission and reflection. That is, the optimizer will first try to reach a 
point in which all $\left|S_{21}\right|$ parameters equals 0.5 and then subsequently expand the difference from the center point.

The Phase 2 design problem is to obtain the full filter functionality. The starting point for Phase 2 are the results of Phase 1 , however, the full filter design problem requires a different objective function. That is, since $\Psi_{1}$ puts an equal weight on transmission and reflection it is therefore likely to lead to designs with $\left|S_{21}\right|$ less than one and $\left|S_{11}\right|$ larger than zero. For the Phase 2 objective function the idea is to decouple transmission and the reflection. This is accomplished by introducing yet a bound variable $\kappa$ that only affects the reflection whereas $\beta$ only affects the transmission. Finally, it is desired that the Phase 2 objective function favors full transmission to that of full reflections. This means that a simple difference between $\kappa$ and $\beta$ does not suffice. However, from band gap optimization [26] it is known that a relative gap measure works very well in practice which leads to the following problem statement for Phase 2:

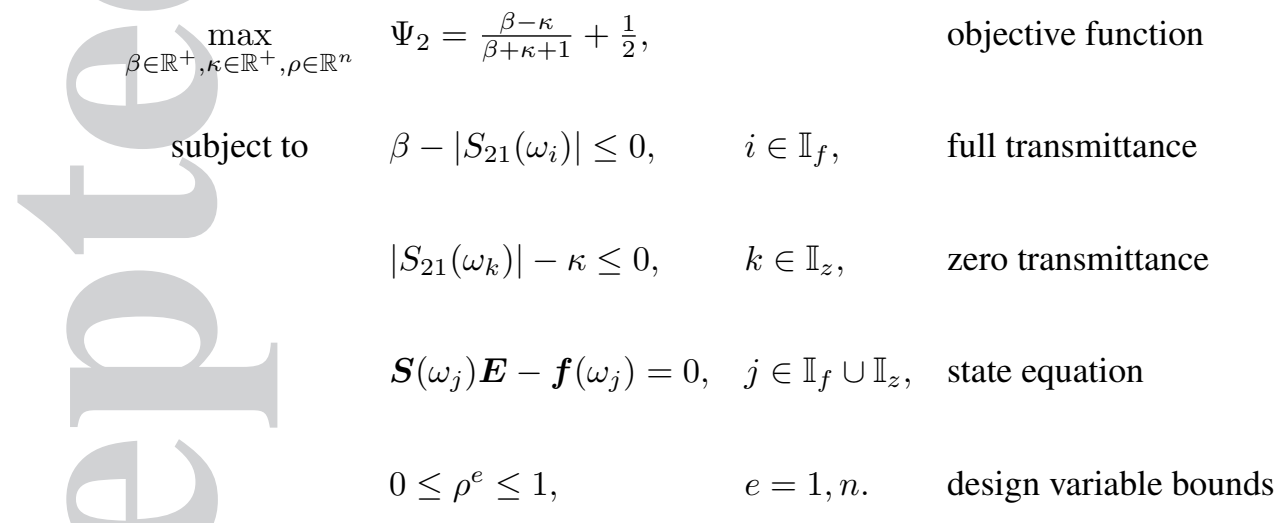

The addition of one to the denominator in $\Psi_{2}$ is needed to avoid zero sensitivities in the cases where either $\beta$ or $\kappa$ is zero. This is similar to the argument used for deriving the RAMP interpolation scheme as presented in [27]. The $1 / 2$ is added to the Phase 2 objective for consistency such that both $\Psi_{1}$ and $\Psi_{2}$ take values between 0 and 1, where 1 refers to optimal performance of the system.

Both the Phase 1 and 2 optimization problems are solved using a gradient based optimization method with sensitivities obtained from the standard adjoint method, see e.g. [28]. The optimization algorithm used is the Method of Moving Asymptotes (MMA) [29, 21].

This article is protected by copyright. All rights reserved. 


\section{DESIGN FILTERING AND CONTINUATION APPROACH}

Solving metallic optimization problems involving wave propagation poses a difficult task in terms of high contrast i.e conductor to air/dielectric [10,12], and many local minima [30]. The used design interpolation and the two phase optimization setup alleviates these issues partially but to make the procedure robust we also include image processing filtering in conjunction with a continuation approach. For small filter radii the standard density filter [31] is used, while for larger radii we apply a standard convolution type density filter to the design problem in terms of a Poisson type equation $[32,33]$, i.e

$$
-\nabla \cdot\left[(2 \sqrt{3} r)^{2} \nabla \tilde{\rho}\right]+\tilde{\rho}=\rho
$$

where $\tilde{\rho}$ is the filtered design field and $r$ corresponds to the radius of influence for the filter. The filter problem is solved with pure Neumann conditions by a linear finite element model and included in the sensitivity calculation by the chain rule cf. [32].

Image filtering naturally introduces gray-scale, i.e. blurred interfaces, in the physical design field.

This is undesirable for several reasons. Firstly, graded materials are expensive to manufacture (if possible) and secondly, resonant structures, such as metallic filters, obtain much of their performance from sharp material interphases. Therefore we suggest the following continuation approach for the length scale parameter $r$. The layout of the scheme is constructed based on our numerical experiments similar to the works of e.g [34] and [35]. For the dimensions used in the reference design of Fig. 4 we have settled on the following filter values and update intervals

$$
r \in\{3.0,1.5,0.75,0.3,0.125\} \mathrm{mm}
$$

The filter radius is updated at every 30th design cycles unless the optimization process converges due to other reasons, i.e. reaching a stationary point. We remark that the optimization process is restarted when the filter radius is updated to acknowledge the non-smooth nature of a sharp parameter change. Note that a filter is included at all times throughout the optimization process. This choice is made to 
ensure a high degree of connectivity in the final design. That is, if the filter is turned off at the final stage in the continuation process, the optimizer can, and often will, lead to designs with free-hanging single elements or small patches thereof.

\section{RESULTS}

In this section we first verify the method and validity of the chosen reference example. Subsequently it is showed how sharper filter characteristics can be obtained on the same design space with more design freedom given. All numerical studies have been carried out on a Linux cluster consisting of 320 nodes each equipped with two Intel Xeon E5-2650 10-core CPUs and 128 GB RAM. We discretized the modelling domain with a total of 737447 tetrahedral elements out of which 204877 are design elements. The mesh is decomposed into 40 parts and the total wall-clock time needed to complete the two phase optimization is approximately 20 hours.

\subsection{Model validation and reference design}

For model validation and later benchmarking, a classical 2-pole insert filter design is chosen with geometry and characteristics as depicted in Fig. 3. The filter design method is still in use today, and we therefore consider it a realistic application example. Formulating the filter characteristics in terms of the optimization problem in Eq. (24) with transmission from $9.8 \mathrm{GHz}$ to $10.4 \mathrm{GHz}$, we have that the discrete list of pass and stop frequencies correspond to that presented in Eq. (19)-(21). To verify that the reference filter indeed is a locally optimal solution this design is used as a start guess for the optimization with the only modification that in order to ensure good design gradients, the void regions were replaced with intermediate material $(\rho=0.3)$. The results of the optimization are seen in Fig. 4, where it is seen that no substantial design changes appeared. This is further confirmed by comparing Fig. $3 b$ to the filter characteristics in Fig. 8(b-c) for the reference design from Fig. 4.

The optimization also validates the code. First of all, the implementation is verified by the fact that the results in Fig. 3(b) are reproduced. Furthermore, it confirms that the optimization procedure 


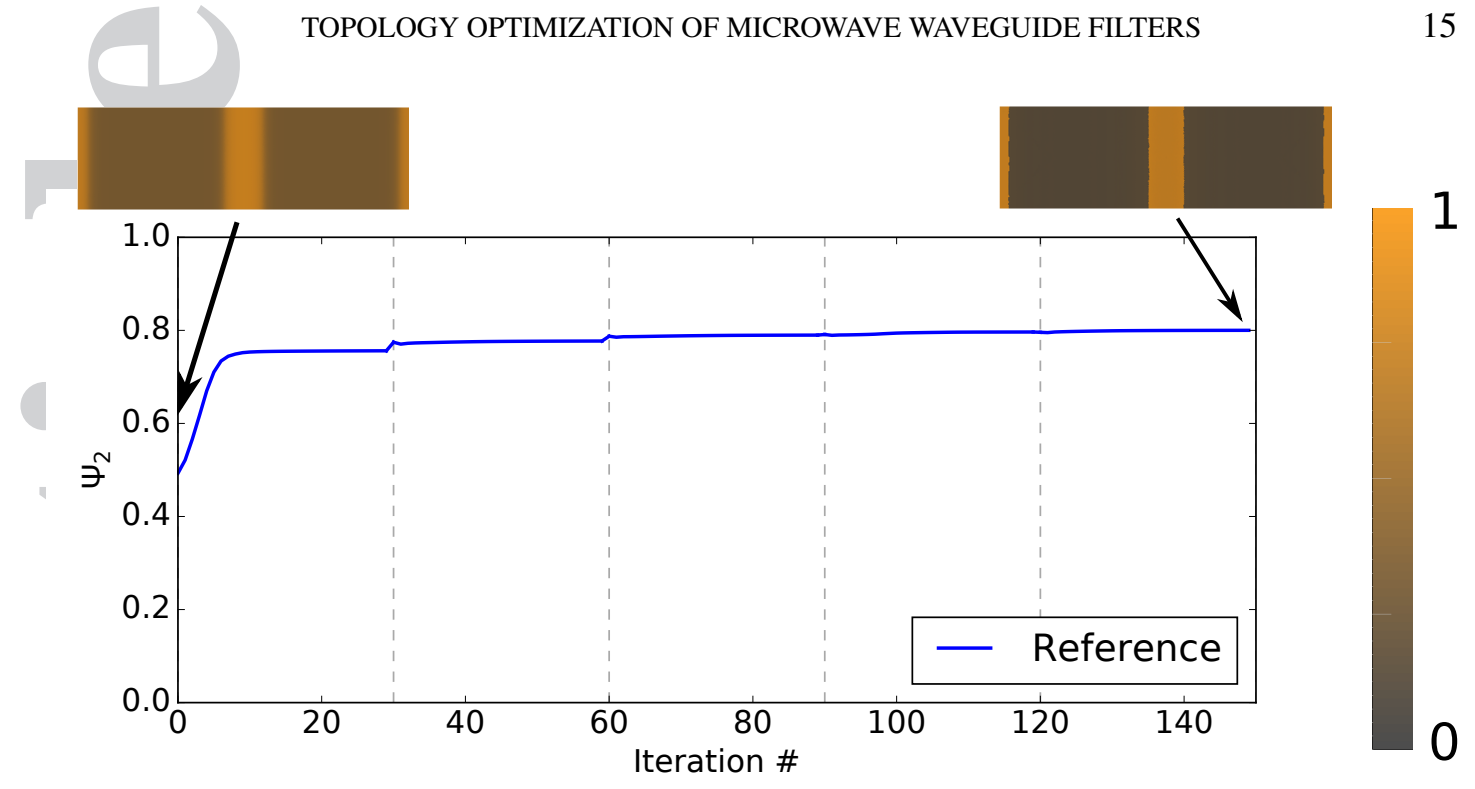

Figure 4. Iteration history including initial guess and final design for the filter which will be used as a reference. The color indicates design interpolation value of $\rho$. The final filter response can be found in Fig. 8(b).

is correctly implemented due to the monotonous behavior of the objective function with respect to iteration number (except for when filter radius is changed due to the continuation approach). The optimization result also confirms that the reference design we want to use as a benchmark is a local minimum with respect to the desired objective function.

Finally note that the reference filter insert design is not designed for a Printed Circuit Board (PCB), i.e. where a metal structure is fabricated on a dielectric background using photo lithographic etching. Using PCBs provides full 2D topological freedom and is included in our numerical model and design methodology. In the following we therefore assume that the structures can be attached to a dielectric with the same permittivity and permeability as air such that direct comparison with the reference design is possible.

\subsection{Topology optimized filter design}

Using the validated code and the reference example as a benchmark, we now show how steeper filter characteristics can be obtained through topology optimization by allowing complete 2D design freedom. The procedure takes outset in a uniform start guess, and is as such not seeded towards any specific structures to begin with.

This article is protected by copyright. All rights reserved. 
8.2.1. Phase 1 - single resonator optimization As described, the first task is to design a number of independent resonators using smaller design volumes. For the pass band filter studied here we choose to start out with three initial resonators. The total design domain has a length of $32.6 \mathrm{~mm}$ equivalent to that of the reference design. We include an air gap of $1 \mathrm{~mm}$ between each of the three resonators to ensure they do not short-circuit when cascaded, which means that each resonator has a design space of $10.2 \mathrm{~mm}$. As target frequencies for each of the Phase 1 resonators we choose three separate resonances inside the passband given by the center frequencies below

$$
f_{c}^{1}=9.8 \mathrm{GHz}, \quad f_{c}^{2}=10.1 \mathrm{GHz}, \quad f_{c}^{3}=10.4 \mathrm{GHz} .
$$

The set of frequencies to maximize and minimize in Eq. (23) for the three different problems are

$$
\begin{aligned}
& \text { given as: } \\
& \qquad \begin{array}{l}
f=f_{c} \times\left\{f_{1}^{z}=1-3 b, f_{2}^{z}=1-2 b, f_{3}^{z} 1-b, f_{4}^{f}=1,\right. \\
\left.\quad f_{5}^{z}=1+b, f_{6}^{z}=1+2 b, f_{7}^{z}=1+3 b\right\}, \\
\mathbb{I}_{f}=\{4\}, \\
\mathbb{I}_{z}=\{1,2,3,5,6,7\},
\end{array}
\end{aligned}
$$

where $f_{c}=f_{c}^{1}, f_{c}^{2}, f_{c}^{3}$ corresponds to each of the three resonator design respectively, and $b=0.08$ is a parameter that defines the trade off between transmission and reflection. We find that giving the reflection frequencies in terms of a fractional bandwidth in general gives better designs than when specifying equidistant frequencies. This is simply explained by the fact that a $100 \mathrm{Mhz}$ difference at two different frequencies does not translate to the same sharpness of a resonator. Likewise, a wavelength dependent scaling of the design domain and filter radii for each resonator could help obtain responses of similar quality, but we have not found it necessary for our optimizations.

The results of the design process and the intermediate steps are seen in Fig. 5. Several trends can be noticed for all three optimizations (the trends are also observed in other design trials we have 
performed): 1) The optimization procedure spend many iterations trying to overcome the state where transmission equals reflection $\left(\Psi_{1}=0.5\right)$, which we interpret as a difficulty of trying to create a structure which gaps reflection and transmission at the desired frequencies. Until then, the algorithm potentially decreases both transmission and reflection at desired frequencies. This is not necessarily a desired route to take for the algorithm, and we hope a future formulation can get rid of this in order to speed up the process and to obtain better resonators at this part of the design stage. 2) The optimizations at different filter radii does not necessarily converge within the given 30 iterations, but we find that better designs are generally obtained by stopping the process prematurely. This is explained by the fact that wave propagation design problems are proned to many (strong) local minima [11], and hence that we wish to terminate the optimization process prematurely before the continuation strategy has reached its end. 3) Topological changes happen several times during the optimization which is a sign of a good degree of design freedom, especially considering how easily metallic microwave problems may get stuck in local minima.

In the end, three soft resonators are found of more or less similar quality based on the objective value. Their filter response is seen in Fig. 8(a). The highest frequency design seems to have the broadest response even though it has the largest design domain measured in wavelengths. This is probably due to the fact that the optimizer had difficulties getting past $\Psi_{1}=0.5$.

8.2.2. Phase 2 - complete filter optimization The three single resonators are now placed next to each other - with a spacing of $1 \mathrm{~mm}$ - and used as starting guess for the actual filter characteristics optimization process, i.e. the Phase 2 problem in (24). Just as for the reference design, the frequencies for the optimization are identical to those presented in Eq. (19)-(21). The objective value at first iteration is $\Psi_{2}=0.502$, which means that an ever so slight gap between transmission and rejection is present, which is crucial for the optimization since we find that the formulation tends to get stuck at $\Psi_{2}=0.5$ as mentioned earlier. However, the Phase 2 objective in Eq. (24) tends to perform significantly better when past this critical point. The outcome of the design procedure is seen in Fig. 6.

This article is protected by copyright. All rights reserved. 


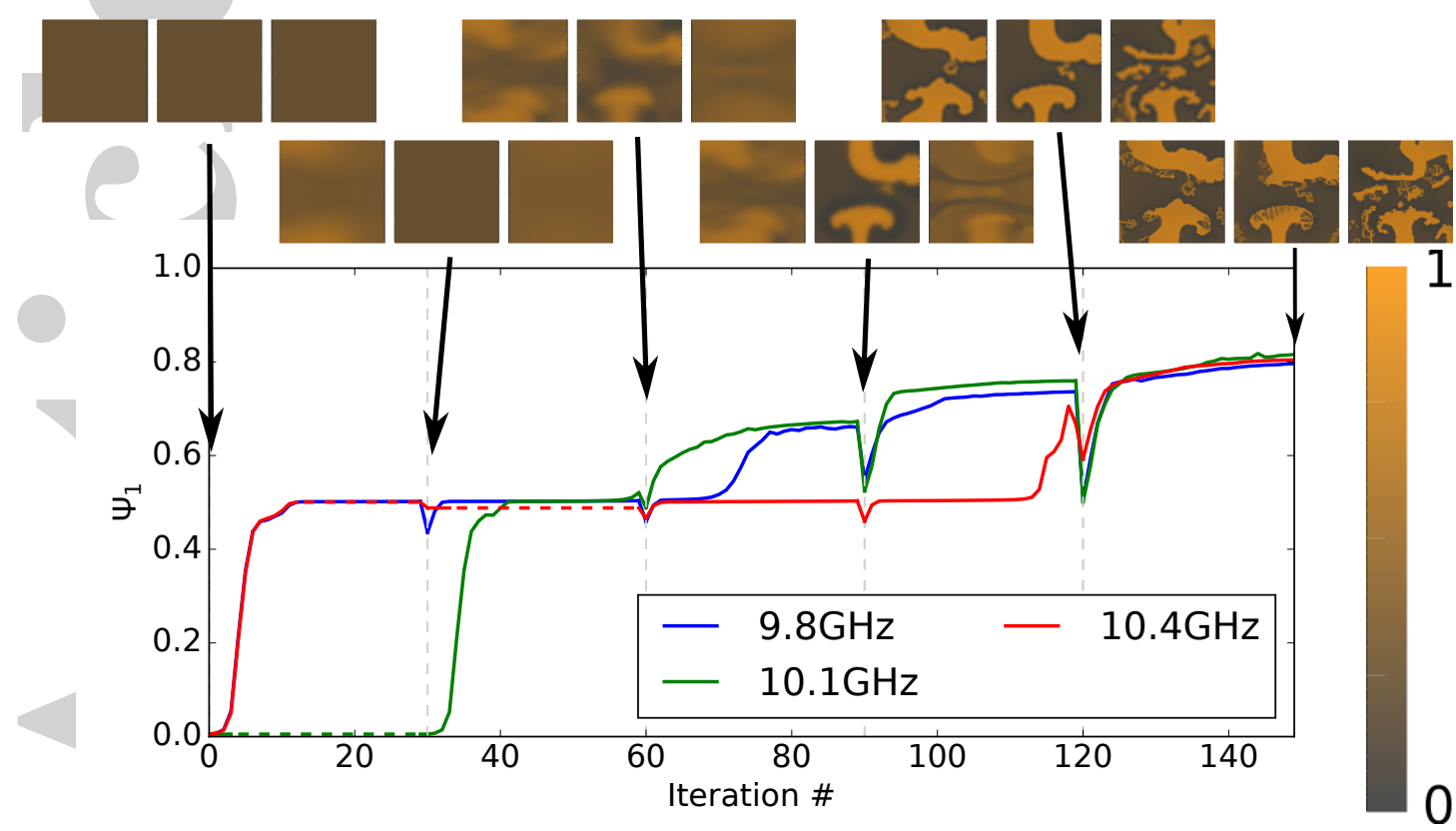

Figure 5. Overview of Phase 1 of the optimization process for the three small, individually optimized, resonators that will make out the start guess for Phase 2. The three intermediate designs are shown above with rising design frequency from left to right. The filter size was decreased every 30th iteration, and the dashed lines indicate that optimization was stopped due to lack of changes in the design.

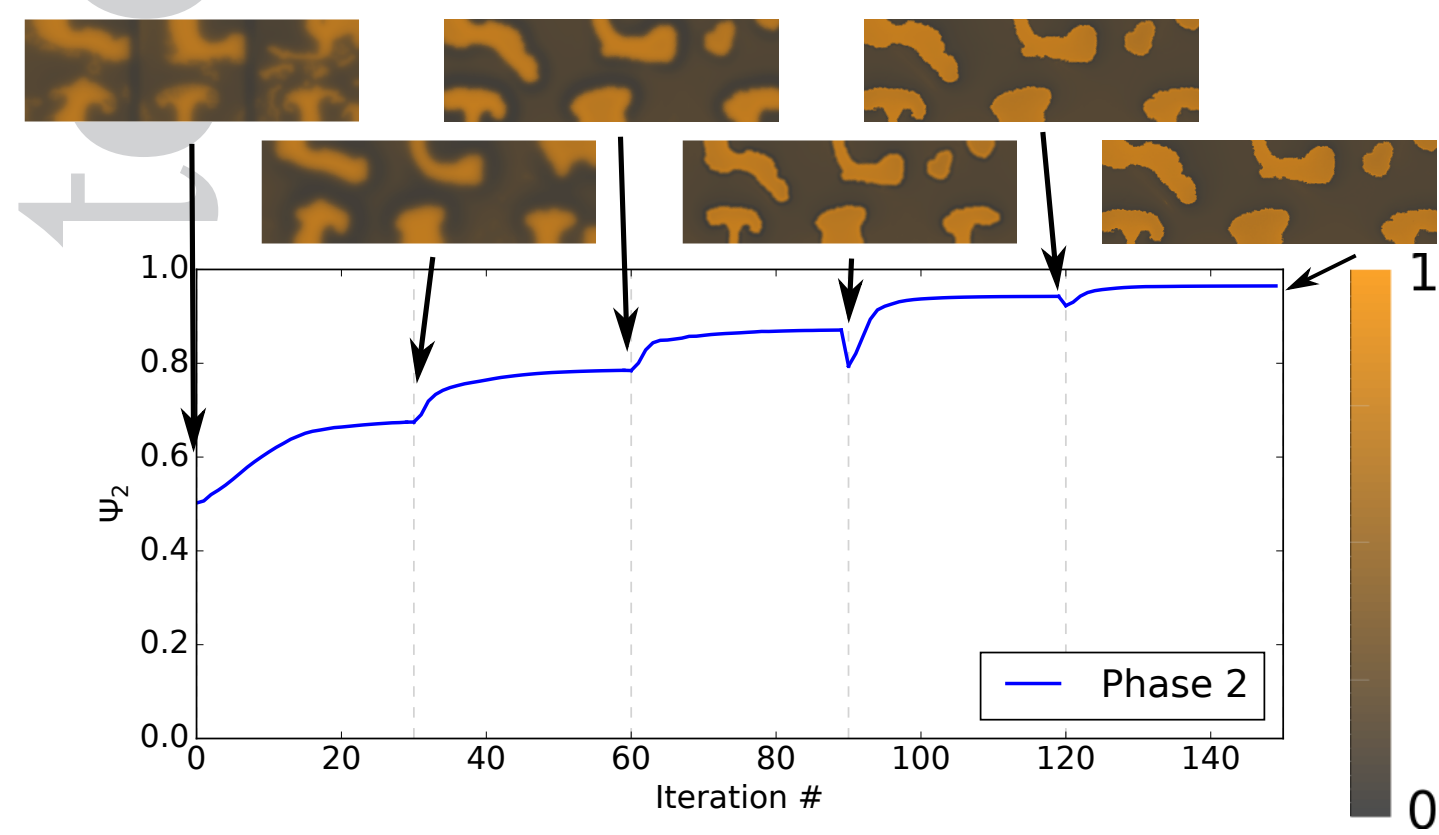

Figure 6. Overview of Phase 2 of the optimization process.

During the first filter radius in the continuation process we observe that small features disappear and that larger features significantly change their shape. Furthermore, several changes in topology takes place during the second step in the continuation process. This clearly indicates how the 


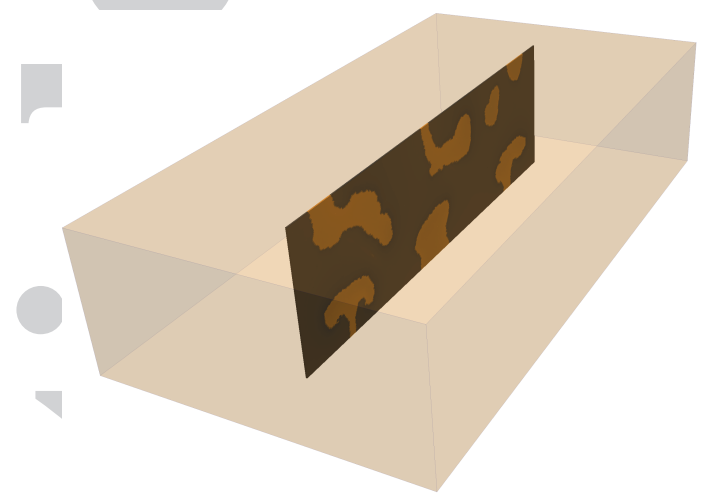

(a) Final design

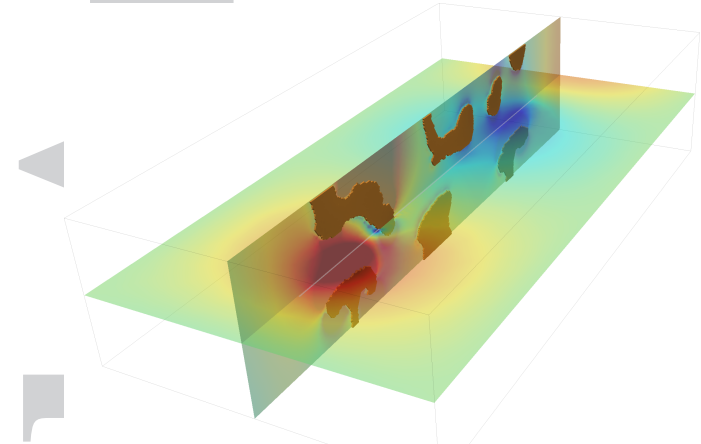

(c) $10.1 \mathrm{GHz}$

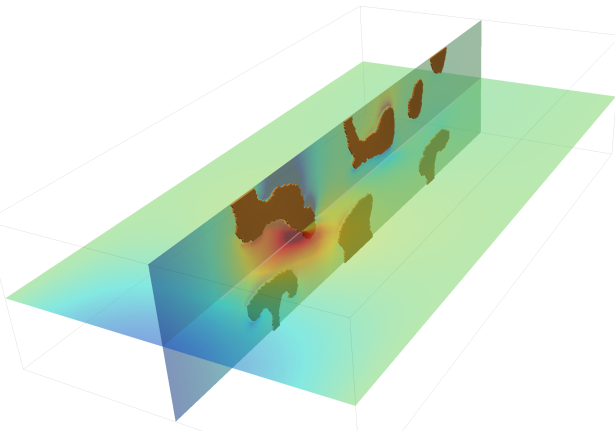

(b) $8 \mathrm{GHz}$

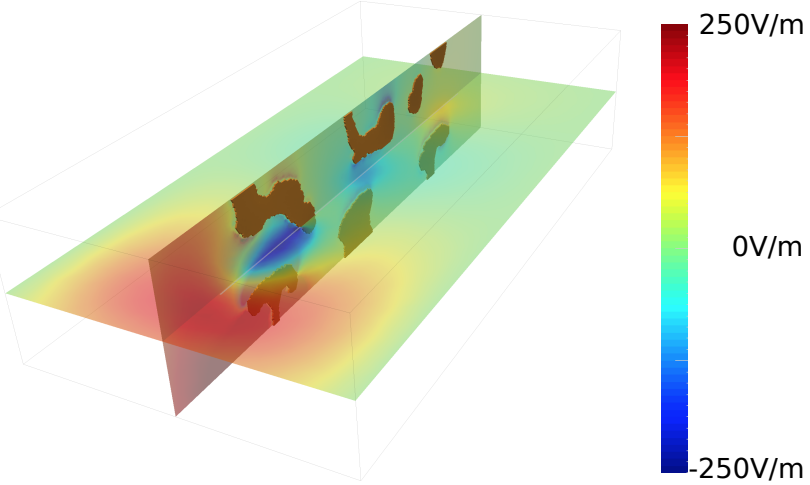

(d) $12 \mathrm{GHz}$

Figure 7. (a) Visualization of how the final design would be sitting in a waveguide in order to act as a filter. The structure is a dielectric material with copper imprint (PCB). The relative dielectric constant is set to 1, but could easily be chosen to fit standard PCB materials. (b-d) Electric field $y$-component for $8 \mathrm{GHz}$, $10.1 \mathrm{GHz}$ and $12 \mathrm{GHz}$ respectively. Since the propagating mode only has a $y$-component, most of the energy is contained here. It is seen how the wave mode is reconstructed at the other side for $10.1 \mathrm{GHz}$, whereas it does not propagate through the filter at the higher/lower frequencies. The input power corresponds to $1 \mathrm{~W}$.

previously obtained resonators in themselves do not provide a good filter response but serve well as a template for creating a larger filter. After this, the topology seems locked and the remaining optimization process constitutes a a shape optimization procedure. The objective of the final design is $\Psi_{2}=0.964$ as compared to the reference design which has an objective of $\Psi_{2}=0.800$. The microwave filter, as it would be situated in an actual waveguide, is depicted in Fig. 7. Electrical field amplitudes at one passband and two stop band frequencies are also shown and clearly demonstrate the filter functionality.

A comparison of the final filter characteristics to the reference filter is seen in Fig. 8(b-c). An insertion loss of $0.5 \mathrm{~dB}$ is seen for both filters. Since the optimized design still contain intermediate design variables, a threshold at $\rho=0.5$ has been introduced after which, the filters are simulated 

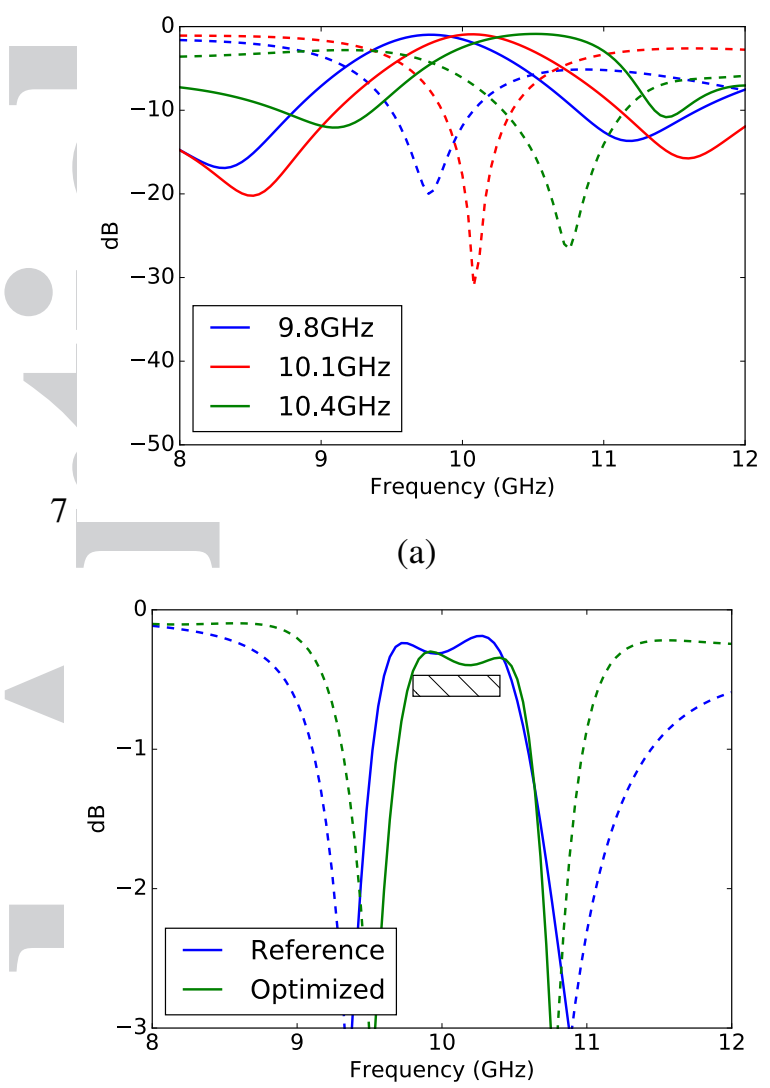

(c)

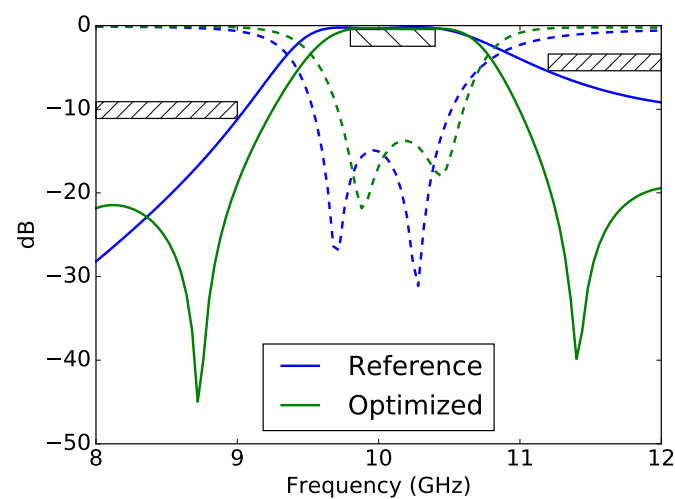

(b)

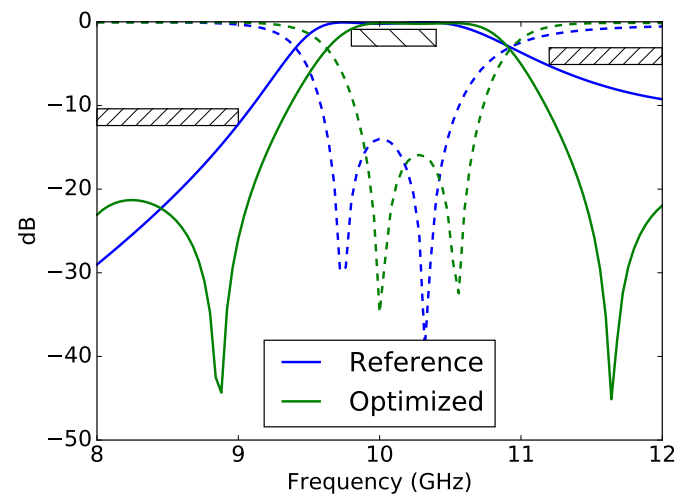

(d)

Figure 8. Filter characteristics for the different optimization results. The solid lines are transmission amplitudes $\left(S_{21}\right)$ and the dashed lines are reflection amplitudes $\left(S_{11}\right)$. (a) Characteristics of the three filters designed in the Phase 1 optimization in Fig. 5. (b) Filter characteristics of the optimized reference filter in Fig. 4 and the freely optimized filter in Fig. 6. (c) Close-up of result in (b). (d) Filter response of a postprocessed version of the filters where the design elements have been exchanged with PEC. All hatched areas indicates the ranges of frequencies defined for either transmission or rejection in the optimization problem.

using a PEC condition. The resulting frequency responses are shown in Fig. 8(d) which confirms the limited influence of our upper bound for material interpolation. It is also noted that a small frequency shift has been introduced by the post processing. This small increase in frequency is attributed to the remaining intermediate design variables and the difference between a real conductor and a PEC.

That is, analogues to vibrations in solids, the addition of viscous damping to a system results in a decrease in the damped natural frequencies.

In comparing the filters, the roll off for the optimized filter is much sharper than for the reference design. This seems to be due to the placement of two anti-resonances/transmission dips at roughly 8.75 GHz and $11.75 \mathrm{GHz}$. Such two resonances are not seen in the reference design, and they cannot This article is protected by copyright. All rights reserved. 
easily be squeezed in using the current design approach without enlarging the design domain. In the transmission band, two poles are still present. One may have hoped for three poles in the transmission band, but in general we find that poles often merge during the optimization process. This is also why it is so important to start with a multipole design, since resonant structures contain strong local minima that are hard to get into as well as hard to get out of. For higher frequencies we see an improvement in the rejection band of $6 \mathrm{~dB}$ or more. For lower frequencies, we see a steeper transition for the optimized design than for the reference (due to the "anti-pole"), but for frequencies around $8 \mathrm{GHz}$, the optimized design actually has a smaller rejection than the reference. This is attributed to the anti-resonance, but since our cost function looked at overall rejection and this increase occurs where the rejection is already quite high, we do not see this as an issue.

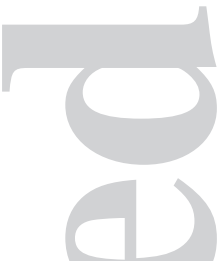

\section{DISCUSSION}

This section is intended to illustrate the necessity of the proposed two phase optimization approach and the filter continuation scheme through a number of numerical experiments. For all cases presented in this Section, the discrete frequency set is identical to that presented in Eq. (19)-(21). However, to further illustrate the effect of the density filter we have added a smaller filter radius to the continuation scheme such that the list now reads

$$
r \in\{3.0,1.5,0.75,0.3,0.125,0.065\} \mathrm{mm}
$$

First, we examine the performance of a pure Phase 1 optimization procedure for two different starting guesses. The optimized design, objective history and filter characteristics can be seen in Fig. 9 for a uniform starting guess with $\rho=0$.3. From Fig. 9(a) it is seen that the design quickly settles and get stuck in a local minima. The subsequent filter continuation merely enhances the contrast between dielectric and conductor, while leaving the overall design topology unchanged and only providing a small increase in performance. The filter characteristics in Fig. 9(b) shows that the 


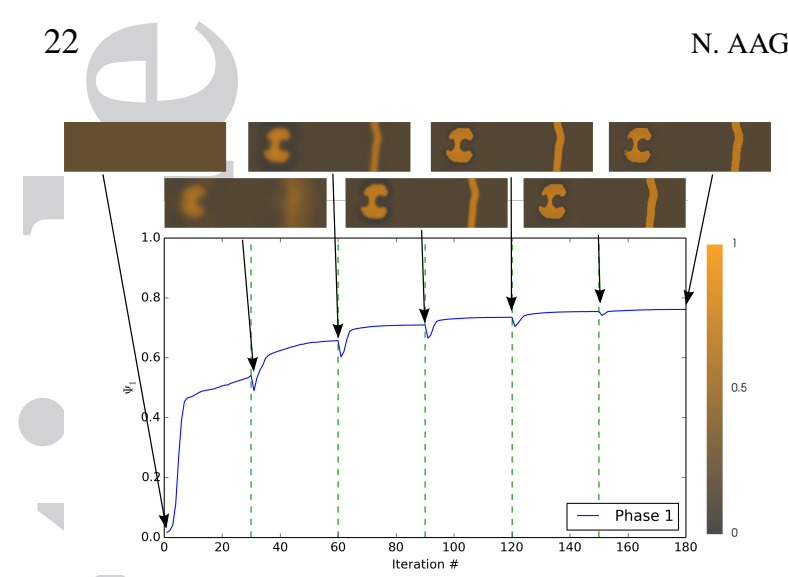

(a)

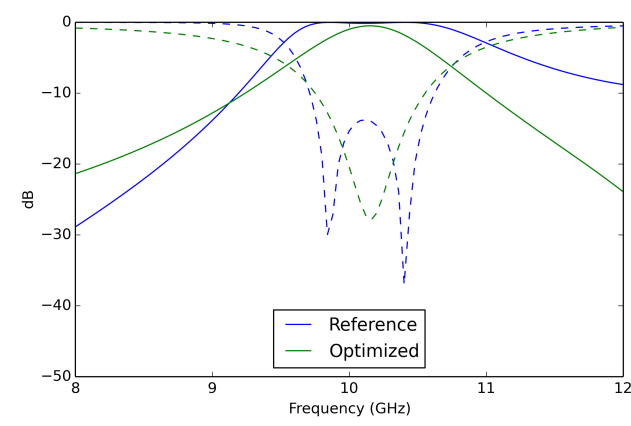

(b)

Figure 9. Result of a pure Phase 1 optimization procedure started from a uniform initial guess with $\rho=0.3$ using the frequency list in Eq. (19)-(21). The objective history and the optimized designs are shown in (a) where the vertical green dotted lines corresponds to changes in filter radius. (b) shows the filter characterics of the final design and the reference. The solid lines are transmission amplitudes $\left(S_{21}\right)$ and the dashed lines

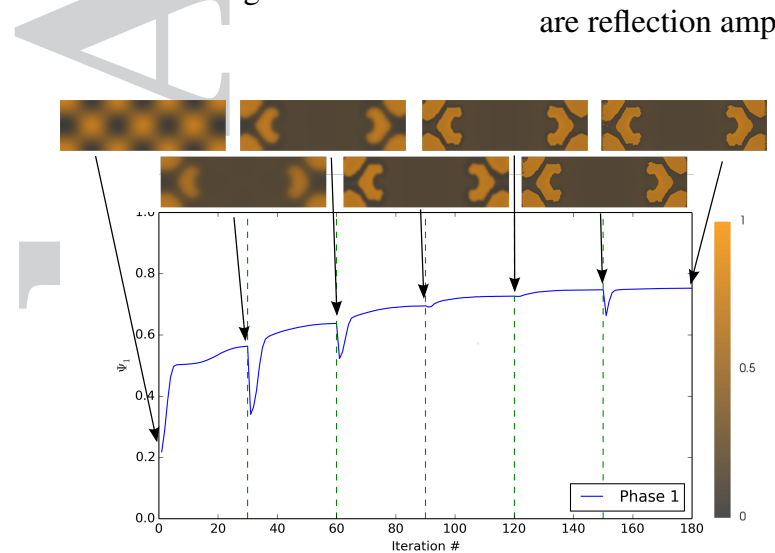

(a)

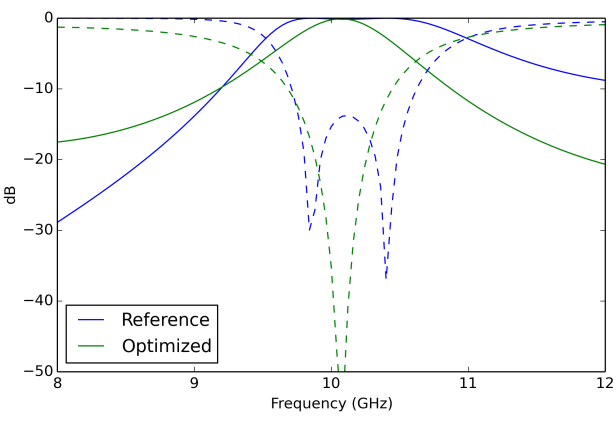

(b)

Figure 10. Result of a pure Phase 1 optimization procedure started from a non-uniform initial guess using Eq. (32) and the frequency list from Eq. (19)-(21). The objective history and the optimized designs are shown in (a) where the vertical green dotted lines corresponds to changes in filter radius. (b) shows the filter characterics of the final optimized design as well as the reference design. The solid lines are transmission amplitudes $\left(S_{21}\right)$ and the dashed lines are reflection amplitudes $\left(S_{11}\right)$.

optimized filter only has a single pole and that the reference design has much better characteristics.

To demonstrate that the designs in Fig. 9 and Fig. 4 are local minima, the pure Phase 1 problem is now solved using a non-uniform starting guess. The initial distribution is given by the following expression

$$
\rho(\boldsymbol{x})=\rho_{\min }+\rho_{\text {ampl. }}\left[\cos \left(\frac{2 \pi m z}{\xi}\right) \cos \left(\frac{2 \pi m y}{\xi}\right)\right]
$$

with $\rho_{\min }=0.4, \rho_{\text {ampl. }}=0.3, \xi=0.03 \mathrm{~m}$ and $m=3$. The non-uniform starting guess can be seen in Fig. 10 which also shows the objective history, the optimized design and the filter characteristics.

From Fig. 10(a) it is observed that the final design is topologically different from that obtained 


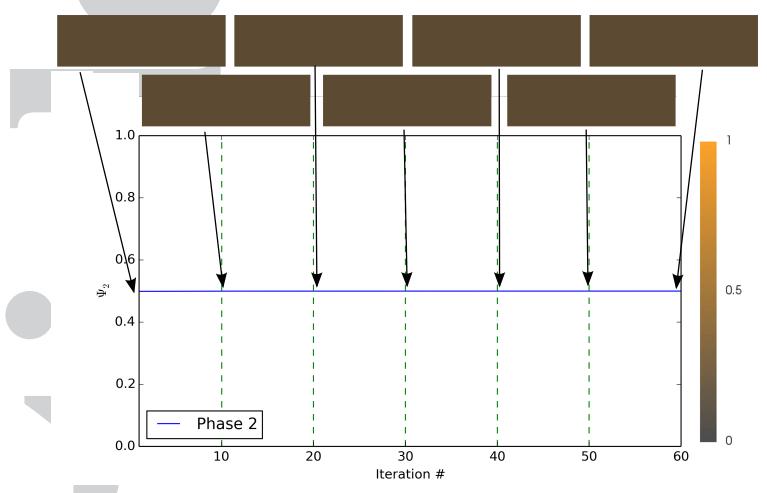

(a)

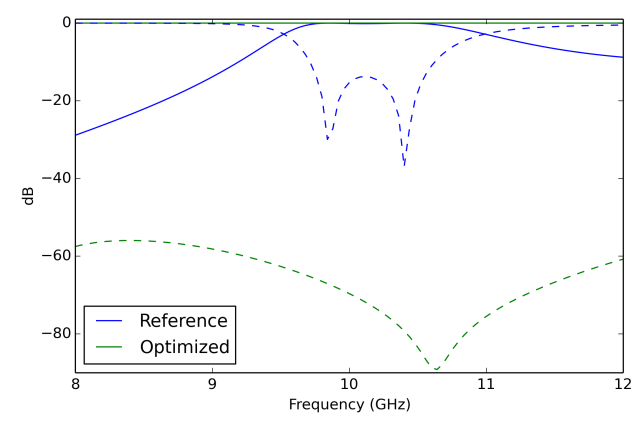

(b)

Figure 11. Result of a pure Phase 2 optimization procedure started from a uniform initial guess with $\rho=0.3$ using the frequency list in Eq. (19)-(21). The objective history and the optimized designs are shown in (a) where the vertical green dotted lines corresponds to changes in filter radius. (b) shows the filter characterics of the final design and the reference. The solid lines are transmission amplitudes $\left(S_{21}\right)$ and the dashed lines are reflection amplitudes $\left(S_{11}\right)$.

with the uniform initial material distribution. Otherwise, similar observations are made. That is, the topology of the design does not change significantly from the iteration 30 and from Fig. 10(b) it is seen that the filter characteristics of the optimized filter only has one pole and that it is outperformed by the reference design.

In the following we study the performance of a pure Phase 2 optimization process using the same two starting guesses as was used for the Phase 1 problems in Fig. 9 and 10, i.e. uniform and using Eq. (32), respectively.

The first results based on a uniform initial distribution of $\rho=0.3$ is shown in Fig. 11. From Fig. 11(a) it is clear that the band gap formulation used in Phase 2 is incapable of producing a functional filter design. This is as explained earlier due to the equal weighting of the full and zero transmission terms in the Phase 2 objective. The poor quality of this optimized design is also evident from the filter characteristic plot in Fig. 11(b). Next, the Phase 2 formulation is used to solve the design based on the initial distribution given by Eq. (32). The resulting optimized design, objective history and filter characteristics are shown in Fig. 12. By examining Fig. 12(a) it is first noted that the Phase 2 optimization process is capable of producing a functional design opposed to the case presneted in Fig. 11. This is because of the initial design, which already has a small initial band gap. However, the same overall tendency is seen again. That is, the design topology is very quickly determined This article is protected by copyright. All rights reserved. 


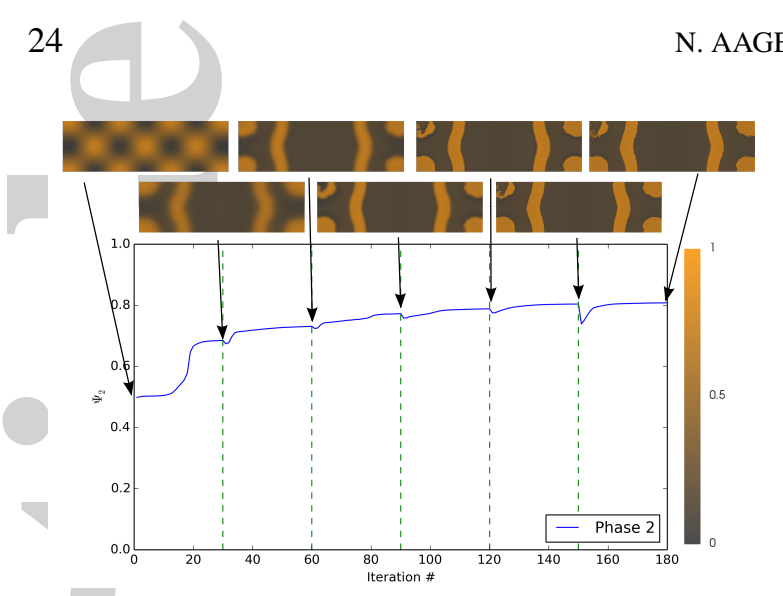

(a)

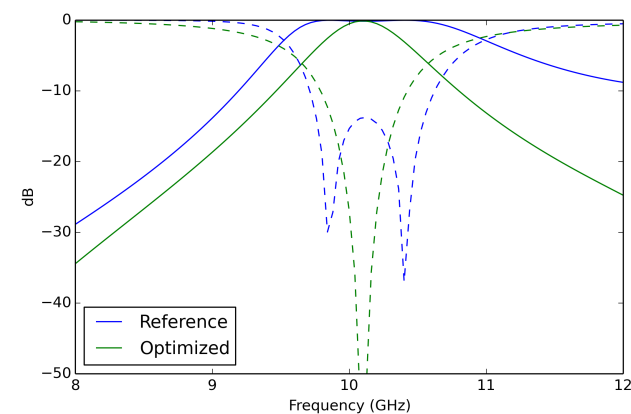

(b)

Figure 12. Result of a pure Phase 2 optimization procedure started from a non-uniform initial guess using Eq. (32) and the frequency list from Eq. (19)-(21). The objective history and the optimized designs are shown in (a) where the vertical green dotted lines corresponds to changes in filter radius. (b) shows the filter characterics of the final optimized design as well as the reference design. The solid lines are transmission amplitudes $\left(S_{21}\right)$ and the dashed lines are reflection amplitudes $\left(S_{11}\right)$.

and the filter characteristic only display one pole, c.f. Fig. 12(b). The performance of the waveguide filter is comparable to those seen in Fig. 9 and 10 although the topologies of all three designs are quite different. For the Phase 2 with a non-uniform starting guess one also observes that the final step in the density filter continuation leads to a decrease in performance, c.f. Fig. 12(a), which we conjecture is due to the many local minima present in the studied design problem and the nonsmooth nature of the continuation scheme. Based on these findings we therefore conjecture that the presented waveguide filter design problem is highly susceptible to local minima and hence motivates the use of the proposed two phase approach.

Finally, the necessity of the filter continuation strategy is illuminated by solving a pure Phase 1 problem using a single fixed filter radius. The design problem is initialized by a uniform starting guess, i.e. $\rho=0.3$, and the filter radius is set to $r=0.065 \mathrm{~mm}$. The optimized design along with objective history and filter characteristics is shown in Fig. 13. The first thing to note in Fig. 13(a) is the high level of small features in the optimized design and that the objective value is significantly lower than that obtained for the designs in Fig. 9, 10 and 12. From the filter characteristics in Fig. 13(b) it is seen that the optimized filter contains multiple poles. However, these additional poles do not enhance the performance of the optimized filter compared to the reference design, e.g. the roll 


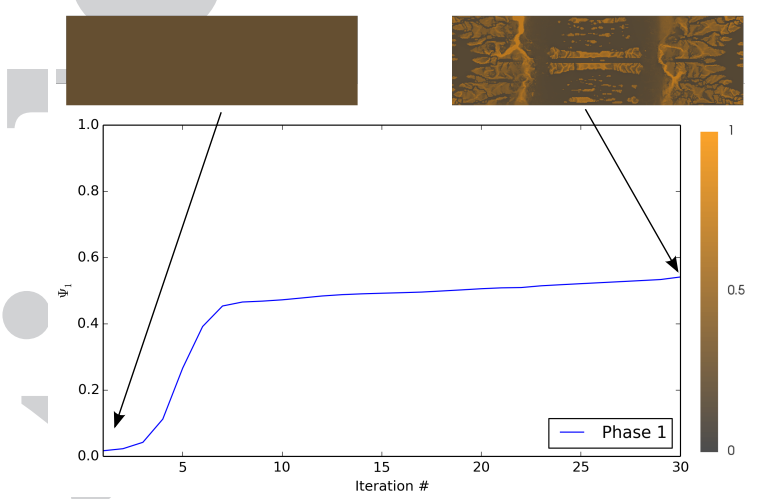

(a)

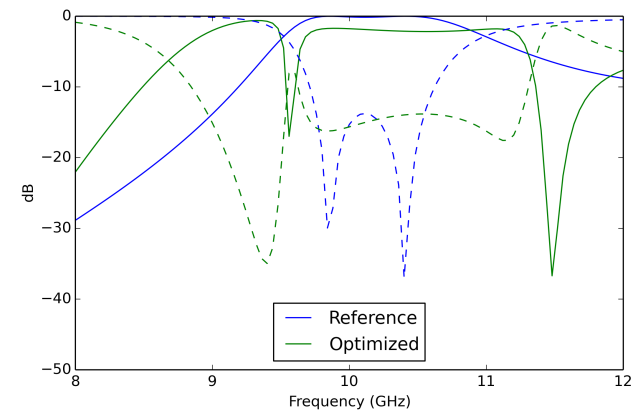

(b)

Figure 13. Result of a pure Phase 1 optimization procedure started from a uniform initial guess with $\rho=0.3$ using only a single filter radius of $r=0.065 \mathrm{~mm}$. The discrete list of frequencies is identical to that in Eq. (19)-(21). The objective history and the optimized designs are shown in (a) and (b) shows the filter characterics of the optimized design as well as the reference design. The solid lines are transmission amplitudes $\left(S_{21}\right)$ and the dashed lines are reflection amplitudes $\left(S_{11}\right)$.

off is less sharp. It is also worth noting that a possible fabrication of the optimized design in Fig. 13 would pose a severe challenge.

\section{OUTLOOK}

For future work, we suggest the following steps to be taken in order to obtain more crisp filter characteristics and wider usability of the design method. First of all, as seen from the iteration history for the Phase 1 design, it is difficult for the algorithm to move past $\Psi_{1}=0.5$. As already mentioned the correct approach would be to use an target eigenfrequency objective, which would overcome this challenge.

It is also worth testing whether or not the reciprocity $\left(S_{21}=S_{12}, S_{11}=S_{22}\right)$ of the problem can be exploited. Using an objective that is averaging two load cases with waves launched from either end of the waveguide, better sensitivities may be obtained over the whole design domain. The idea behind this approach is similar to how the problems in topology optimized antenna design are inverted by first considering radiation and then illumination [11].

For filter design with more strict control of the response, asymptotic wave expansion (AWE)[20, 36] could help increasing solution performance and computational effort by providing a way to This article is protected by copyright. All rights reserved. 
obtain a continuous filter response curve instead of the relatively low set of discrete data points we are using at the moment. AWE would furthermore allow more detailed control of the filter characteristics, but at the cost of more constraints and hence a harder optimization problem.

Opposed to the AWE approach it would also be interesting to try out differently spaced sets of discrete frequencies and to perform a systematic study of the influence of this choice on the proposed design methodology.
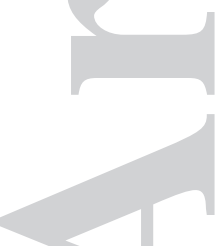

\section{CONCLUSIONS}

We have demonstrated a topology optimization methodology capapble of designing metallic waveguide filters with improved performance as compared to a benchmark example. The approach is implemented using the finite element method (FEM) and is divided into two design phases in order to obtain the strong resonances required for obtaining a filter with high transmission and rejection characteristics. The obtained design does not bear resemblance with any earlier design and thus indicates the potential for rethinking traditional filter design approaches for microwave engineering. We believe that this proof-of-concept is the first step towards having topology optimized microwave filters in engineering applications.

\section{ACKNOWLEDGEMENTS}

This work was funded by the Villum Foundation through the NextTop project and the Danish National Advanced Technology Foundation through the grant Wireless Coupling in Small Autonomous Apparatus (www.hoejteknologifonden.dk). The authors would like thank to Vitaliy Zhurbenko and the DTU TopOpt group for valuable insight and many fruitful discussion.

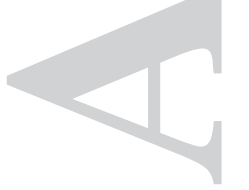

REFERENCES

1. Pozar DM. Microwave Engineering. 3rd edn., John Wiley \& Sons, Ltd., 2005.

This article is protected by copyright. All rights reserved. 
2. Byun JK, Park IH. Design of dielectric waveguide filters using topology optimization technique. IEEE transactions on magnetics 2007; 43(4):1573-1576.

3. Khalil H, Assadihaghi A, Bila S, Baillargeat D, Aubourg M, Verdeyme S, Puech J, Lapierre L. Topology gradient optimization in 2-D and 3-D for the design of microwave components. Microwave and optical technology letters 2008; 50(10):895-896.

4. Khalil H, Delhote N, Bila S, Aubourg M, Verdeyme S, Puech J, Lapierre L, Delage C, Chartier T. Topology optimization applied to the design of a dual-mode filter including a dielectric resonator. Microwave Symposium Digest, 2008 IEEE MTT-S International, 2008; 1381-1384.

5. Khalil H, Bila S, Aubourg M, Baillargeat D, Verdeyme S, Puech J, Lapierre L, Delage C, Chartier T. Topology optimization of microwave filters including dielectric resonators. Microwave Conference, 2009. EuMC 2009. European, 2009; 687-690.

6. Choi NS, Kim DH, Lee HB, Byun JK. Topology Optimization of Dielectric Resonator in 3-D Waveguide Structure Considering Higher Mode Incidence. Magnetics, IEEE Transactions on feb 2012; 48(2):559-562.

7. Delhote N, Bila S, Baillargeat D, Chartier T, Verdeyme S. Advanced design and manufacturing of microwave components based on shape optimization and ceramic stereolithography process. 2008 IEEE MTT-S International

Microwave Workshop Series IMWS on Art of Miniaturizing RF and Microwave Passive Components - Proceeding $2011 ;: 15-18$.

8. Jensen JS, Sigmund O. Topology optimization of photonic crystal structures: a high-bandwidth low-loss T-junction waveguide. Journal of the Optical Society of America B 2005; 22(6):1191.

9. Matzen R, Jensen JS, Sigmund O. Topology optimization for transient response of photonic crystal structures. Journal of the Optical Society of America B 2010; 27(10):2040.

10. Aage N, Mortensen NA, Sigmund O. Topology optimization of metallic devices for microwave applications. International Journal for Numerical Methods in Engineering 2010; 83(2):228-248.

11. Aage N. Topology optimization of radio frequency and microwave structures. Ph.d. dissertation, Techanical University of Denmark 2011

12. Hassan E, Wadbro E, Berggren M. Topology Optimization of UWB Monopole Antennas. Technical Report 2013.

13. Hassan E, Wadbro E, Berggren M. Topology optimization of metallic antennas. IEEE Transactions on Antennas and Propagation 2014; 62(X):2488-2500.

14. Ouedraogo RO, Rothwell EJ, Diaz AR, Fuchi K, Tang J. Waveguide band-stop filter design using optimized pixelated inserts. Microwave and optical technology letters 2013; 55(1).

15. Assadihaghi A, Bila S, Durousseau C, Baillargeat D, Aubourg M, Verdeyme S, Rochette M, Puech J, Lapierre L. Design of Microwave Components using Topology Gradient Optimization. Microwave Conference, 2006. 36th European, 2006; 462-465.

16. Erentok A, Sigmund O. Topology optimization of sub-wavelength antennas. IEEE Transactions on Antennas and Propagation 2011; 59(1):58-69.

This article is protected by copyright. All rights reserved. 
17. Nomura T, Ohkado M, Schmalenberg P, Lee J, Ahmed O, Bakr M. Topology Optimization Method for Microstrips using Boundary Condition Representation. Proceedings of the 43rd European Microwave Conference 2013; :632635.

18. Hassan E, Noreland D, Augustine R, Wadbro E, Berggren M. Topology Optimization of Planar Antennas for Wideband Near-Field Coupling. IEEE Transactions on Antennas and Propagation 2015; 63(9):4208-4213.

19. Balanis CA. Advanced Engineering Electromagnetics. 2nd edn., CourseSmart Series, Wiley, 2012.

20. Jin JM. The Finite Element Method in Electromagnetics. A Wiley-Interscience publication, Wiley, 2002.

21. Aage N, Lazarov BS. Parallel framework for topology optimization using the method of moving asymptotes. Structural and Multidisciplinary Optimization 2013; 47(4):493-505.

22. Karypis G, Kumar V. A Fast and Highly Quality Multilevel Scheme for Partitioning Irregular Graphs. SIAM Journal on Scientific Computing 1999; 20(1):359-392.

23. Amestoy P, Duff I, L'Excellent J. Multifrontal parallel distributed symmetric and unsymmetric solvers. Computer Methods in Applied Mechanics and Engineering 2000; 184(2-4):501-520.

24. Bendsoe MP, Sigmund O. Topology Optimization: Theory, Methods and Applications. Springer Verlag, Berlin Heidelberg, 2004.

25. Jensen JS, Pedersen NL. On maximal eigenfrequency separation in two-material structures: The 1D and 2D scalar cases. Journal of Sound and Vibration 2006; 289(4-5):967-986.

26. Sigmund O, Jensen JS. Systematic design of phononic band-gap materials and structures by topology optimization. Philosophical transactions. Series A, Mathematical, physical, and engineering sciences 2003; 361(May):10011019.

27. Stolpe M, Svanberg K. An alternative interpolation scheme for minimum compliance topology optimization. Structural and Multidisciplinary Optimization 2001; 22(2):116-124.

28. Christensen PW, Klarbring A. An Introduction to Structural Optimization. Springer, 2009.

29. Svanberg K. The method of moving asymptotes - a new method for structural optimization. International Journal for Numerical Methods in Engineering 1987; 24(June 1986):359-373.

30. Diaz AR, Sigmund O. A topology optimization method for design of negative permeability metamaterials. Structural and Multidisciplinary Optimization 2010; 41:163-177.

31. Bourdin B. Filters in topology optimization. International Journal for Numerical Methods in Engineering 2001; 50(December 1999):2143-2158.

32. Lazarov, Boyan Stefanov and Sigmund O. Filters in topology optimization based on Helmholtz-type differential equations. International Journal for Numerical Methods in Engineering 2010; :1885-1891.

33. Kawamoto A, Matsumori T, Yamasaki S, Nomura T, Kondoh T, Nishiwaki S. Heaviside projection based topology optimization by a PDE-filtered scalar function. Structural and Multidisciplinary Optimization 2011; 44:19-24.

34. Alexandersen J, Sigmund O, Aage N. Large scale three-dimensional topology optimisation of heat sinks cooled by natural convection. International Journal of Heat and Mass Transfer 2016; 100:876-891.

This article is protected by copyright. All rights reserved. 
35. Wang F, Lazarov BS, Sigmund O. On projection methods, convergence and robust formulations in topology optimization. Structural and Multidisciplinary Optimization 2011; 43:767-784.

36. Jensen JS. Topology optimization of dynamics problems with Pade approximants. Proceedings of the 2011 American Control Conference 2007; (April):1605-1630.
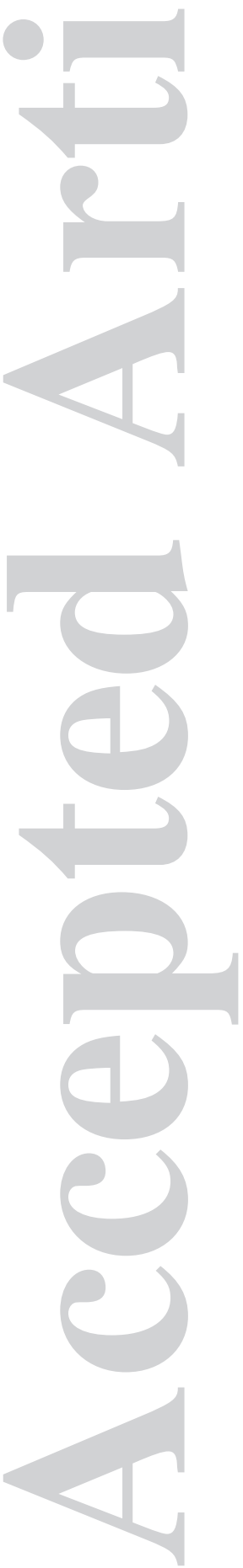

This article is protected by copyright. All rights reserved. 